\title{
MATERIALES CERÁMICOS COLONIALES EN ANTIGUYOC (PUNA DE JUJUY, ARGENTINA): APROXIMACIÓN A SU PRODUCCIÓN, CIRCULACIÓN Y USO
}

\author{
COLONIAL CERAMIC MATERIALS IN ANTIGUYOC \\ (JUJUY PUNA, ARGENTINA): AN APPROACH TO THEIR PRODUCTION, \\ CIRCULATION AND USE
}

\author{
M. Josefina Pérez Pieroni* y Marco N. Giusta**
}

\begin{abstract}
Presentamos los resultados obtenidos hasta la fecha del estudio de evidencias materiales y documentación histórica de Antiguyoc, una localidad de la Puna de Jujuy vinculada a la minería del oro de época tardocolonial. El objetivo particular del trabajo consistió en aportar conocimientos acerca de la organización de la manufactura y la circulación de materiales cerámicos, en el marco de objetivos más amplios que consisten en el estudio del devenir histórico del lugar, de la vida cotidiana de las personas que lo habitaron y de las actividades que llevaron a cabo.

El estudio presentado hace hincapié en parte de los materiales cerámicos recuperados en superficie del sitio Antiguyoc 1, el que funcionó desde fines del siglo XVIII como asiento minero y viceparroquia. A su vez incorporamos datos provenientes de fuentes escritas que si bien refieren a otros espacios, son lugares próximos al mencionado sitio y que en la época analizada formaban parte de la jurisdicción de Antiguyoc.
\end{abstract}

Palabras claves: Cerámica, Puna de Jujuy, siglo XVIII, arqueología histórica.

In this paper we present the results obtained to date of the study of material evidence and historical documentation of Antiguyoc, a town in Puna de Jujuy linked to colonial gold mining. The particular objective was to provide knowledge about the organization of the manufacture and circulation of ceramic materials in Antiguyoc, within the framework of broader objectives that consist in the study of the historical development of the place, of people's daily life and the activities they carried out.

The study emphasizes part of the ceramic materials recovered on the surface of the Antiguyoc 1 site, which functioned since the end of the 18th century as a mining seat and vice parish. At the same time, we incorporate data from written sources that, although they refer to other spaces, are places close to the mentioned site and that at the time analyzed were part of the jurisdiction of Antiguyoc.

Key words: Pottery, Puna de Jujuy, $18^{\text {th }}$ century, historical archaeology.

\section{Introducción}

Presentamos resultados obtenidos hasta la fecha del estudio de evidencias materiales y documentación histórica de Antiguyoc, una localidad de la Puna jujeña vinculada a la minería del oro de época colonial (Figura 1). Los objetivos generales que guían nuestras tareas son: aportar al conocimiento del devenir histórico del lugar, de la vida cotidiana de las personas que lo habitaron y de las actividades que llevaron adelante. Como parte de este último ítem, nos interesa en particular contribuir a la comprensión de la organización de la manufactura y la circulación de materiales cerámicos, tanto en
Antiguyoc como en otros centros mineros coloniales de la Puna de Jujuy.

La instalación de asentamientos hispánicos en Antiguyoc, habría cobrado relevancia hacia el último cuarto del siglo XVIII, en consonancia con factores como el repunte de la actividad minera, el desarrollo de emprendimientos ganaderos y nuevas segmentaciones eclesiásticas del espacio puneño (Gil Montero 2004). Recordemos que la minería, a pesar de su modesta escala, desde inicios del siglo anterior reunía una significativa población en torno a los asientos mineros. Es en este contexto que en momentos tardocoloniales se desarrolló en Antiguyoc un emprendimiento minero-ganadero, y

\footnotetext{
* Consejo Nacional de Investigaciones Científicas y Técnicas - Instituto Superior de Estudios Sociales - Instituto de Arqueología y Museo, Facultad de Ciencias Naturales e IML, Universidad Nacional de Tucumán. Tucumán, Argentina. Correo electrónico: josefinaperezp@gmail.com

** Consejo Nacional de Investigaciones Científicas y Técnicas - Instituto Superior de Estudios Sociales - Instituto de Arqueología y Museo, Facultad de Ciencias Naturales e IML, Universidad Nacional de Tucumán. Tucumán, Argentina. Correo electrónico: giustamarco@yahoo.com.ar
} 


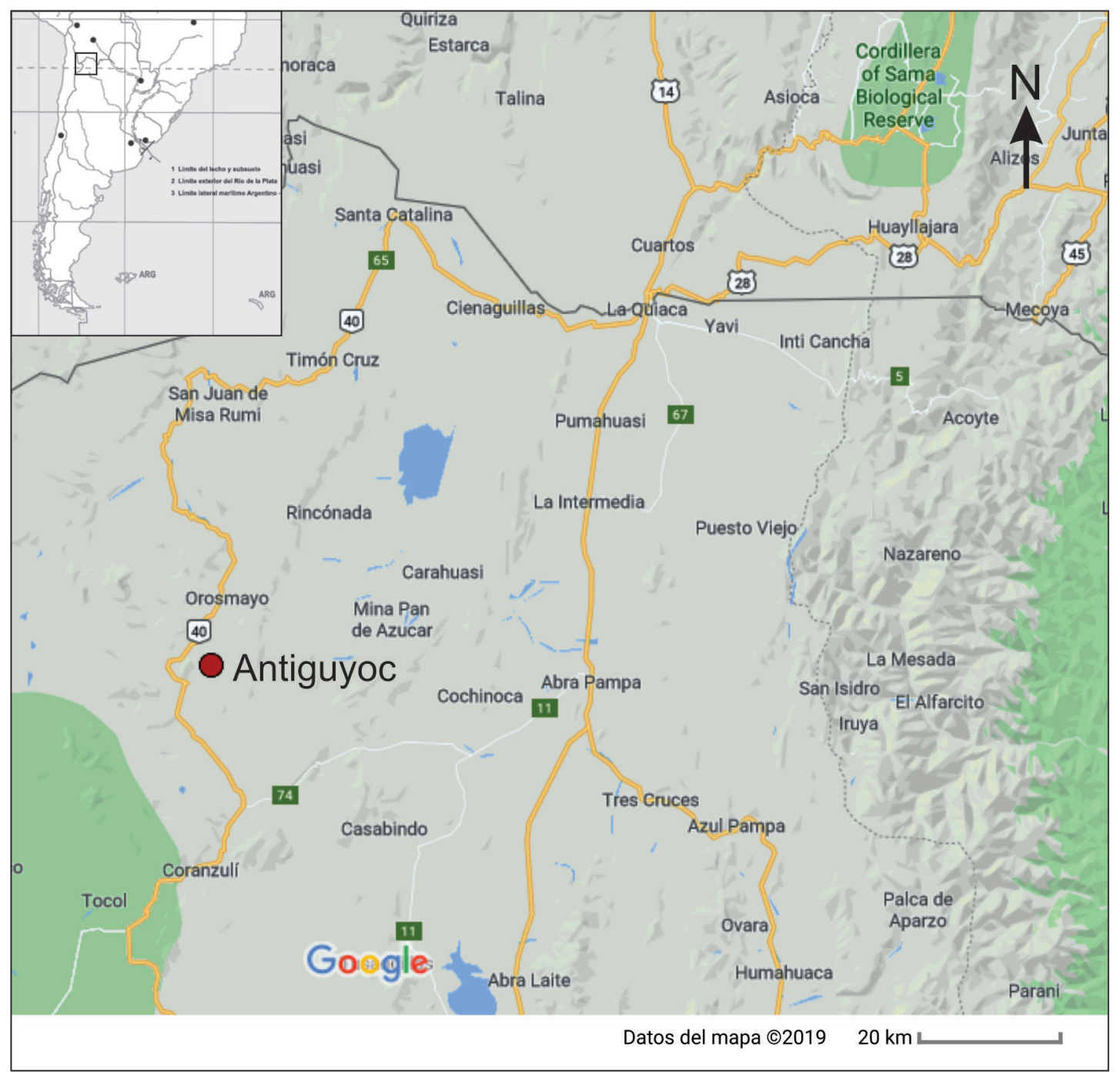

Figura 1. Ubicación de Antiguyoc. Elaborado sobre mapa de Google Maps.

a su vez en 1777 adquirió estatus de viceparroquia dependiente del curato de Rinconada (Angiorama et al. 2018a; Gil Montero 2004; Ulloa 2005; Vergara 1942).

\section{Una localidad, varios lugares}

La localidad de Antiguyoc se encuentra ubicada en la vertiente occidental de la Sierra de Carahuasi (departamento Rinconada), en un ámbito estructurado por la cuenca del río Ajedrez, único cauce permanente de la zona, el que recibe aportes de otros cauces menores, como los que discurren por las quebradas de Antiguyoc y del Maray. A partir de nuestras investigaciones, hemos planteado que el avance hispánico en este sector se tradujo en al menos dos asentamientos principales, a los que arqueológicamente hemos denominado Antiguyoc 1 (en adelante AN1) y Quebrada del Maray 4 (en adelante QM4) (Angiorama et al. 2018a), distanciados entre sí $2 \mathrm{~km}$ lineales (Figura 2). Hacia 1776 se asentaron allí unos pocos españoles (entre ellos Fernando Dávalos, el propietario de las tierras en las que se emplazan los sitios) y población indígena y mestiza que trabajaba en las minas y en la ganadería. La vivienda de Dávalos estuvo ubicada en QM4, donde también contaba con una cancha para matanza de ganado, mientras que AN1 se 
transformó en el asentamiento más poblado, sede de la viceparroquia de Nuestra Señora del Carmen de Antiguyoc (1777-1824) y en el asiento minero principal (Giusta 2019). Además sabemos por la documentación escrita y por nuestras propias investigaciones arqueológicas, que también existieron otros espacios ocupados por población indígena (y posiblemente también mestiza) que constituían sus domicilios o estancias de pastoreo (sensu Tomasi 2010), conformándose así el patrón de "residencias múltiples" planteado por Gil Montero $(1997,2004)$ y que aún persiste en esta y otras áreas de puna del NOA, como se ha comprobado etnográficamente (Delfino 2001; Göbel 2002; Tomasi 2010, entre otros). Uno de estos lugares, dentro de la localidad de Antiguyoc, estaba ubicado en la quebrada de Toronsaire, emplazada a unos $11 \mathrm{~km}$ de distancia aproximadamente respecto de Antiguyoc 1 (ver Figura 2). Su curso de agua, al confluir con el río Ajedrez, da lugar al río Orosmayo.

En este trabajo, los lugares mencionados están representados desde diferentes aproximaciones: AN1 es el sitio del que provienen los materiales cerámicos analizados, mientras que QM4 y la Quebrada de Toronsaire son los espacios a los que se hace referencia en las fuentes escritas que hemos considerado. En este sentido, las diferentes líneas de evidencias nos acercan a tres contextos distintos contemporáneos dentro de una misma localidad o jurisdicción: una vivienda hispana (QM4), una vivienda rural indígena (Toronsaire) y un asiento minero (AN1) con población diversa (españoles, indígenas y mestizos).

El sitio arqueológico AN1 es un caserío constituido por construcciones en piedra, con estructuras próximas entre sí que conforman un núcleo central, en el que destacan una iglesia con su patio posterior delimitado por muros altos en "L", la torre de campanario y un espacio despejado al frente, a modo de "plaza" (Figura 3). Al alejarse de la iglesia, las construcciones se encuentran más distanciadas, y a 200 metros de la misma se ubica un cementerio. Tanto la iglesia como el cementerio son espacios en los que se han registrado reocupaciones y reutilizaciones de variada longitud e intensidad en momentos recientes (Angiorama et al. 2018a).

\section{Materiales y metodología}

Los trabajos realizados hasta la fecha, y de los que presentamos aquí algunos avances, involucraron tareas de campo y gabinete. En ambos sitios (AN1 y QM4) llevamos adelante la planimetría, registro arquitectónico y recolección de materiales superficiales. Se definieron conjuntos de estructuras asociadas (denominados conjuntos arquitectónicos, en adelante CA), relevando sus características en planillas diseñadas para tal fin y registrando los materiales asociados a cada uno. En la etapa de gabinete, respecto de los materiales recolectados, profundizamos el estudio de los fragmentos cerámicos, que incluyen cerámicas rojas, lozas, porcelana y gres.

Como hemos señalado, la localidad de Antiguyoc estaba ocupada en diferentes espacios. El material cerámico analizado para este trabajo corresponde a los fragmentos recolectados superficialmente en 10 de los 20 CA con construcciones habitacionales definidos de AN1 y del espacio abierto a modo de plaza frente a la iglesia (Tabla 1), que constituye el material procesado hasta el momento (812 de 1.037 fragmentos recolectados hasta la fecha). El resto de los materiales cerámicos y no cerámicos aún se encuentra bajo estudio. La muestra se analizó macroscópicamente, teniendo en cuenta los atributos sistematizados para nuestros trabajos previos con materiales prehispánicos y coloniales de la cuenca sur de Pozuelos (departamento Rinconada) y el área de Santa Catalina, en el departamento homónimo (Pérez Pieroni 2015a, 2018). Estos incluyen definición de la morfología (reconstruibles en ocasiones a partir de los bordes, siguiendo el sistema clasificatorio de Balfet et al. 1992 y el elaborado en la Puna de Jujuy por Menacho, en Zaburlín 2014), atributos morfológicos de bordes, bases y asas, registro de macrotrazas de modelado (García Roselló y Calvo Trías 2013), de acabados de superficie, la observación submacroscópica de las pastas (en lupa binocular Motic DM 39C - 20 a 40X) y su clasificación en grupos de pasta definidos previamente para el área (Pérez Pieroni 2015b).

Respecto de los documentos analizados, dentro del corpus documental disponible que refiere a Antiguyoc (Giusta 2019), en este trabajo seleccionamos y nos referiremos a aquellos que mencionan artefactos utilizados como recipientes, en especial de material cerámico. Dos de ellos refieren a las posesiones que se hallaban en la hacienda española ubicada en Quebrada del Maray 4, mientras que en el otro se describe una vivienda con los objetos hallados en su interior ubicada en la quebrada de Toronsaire. 


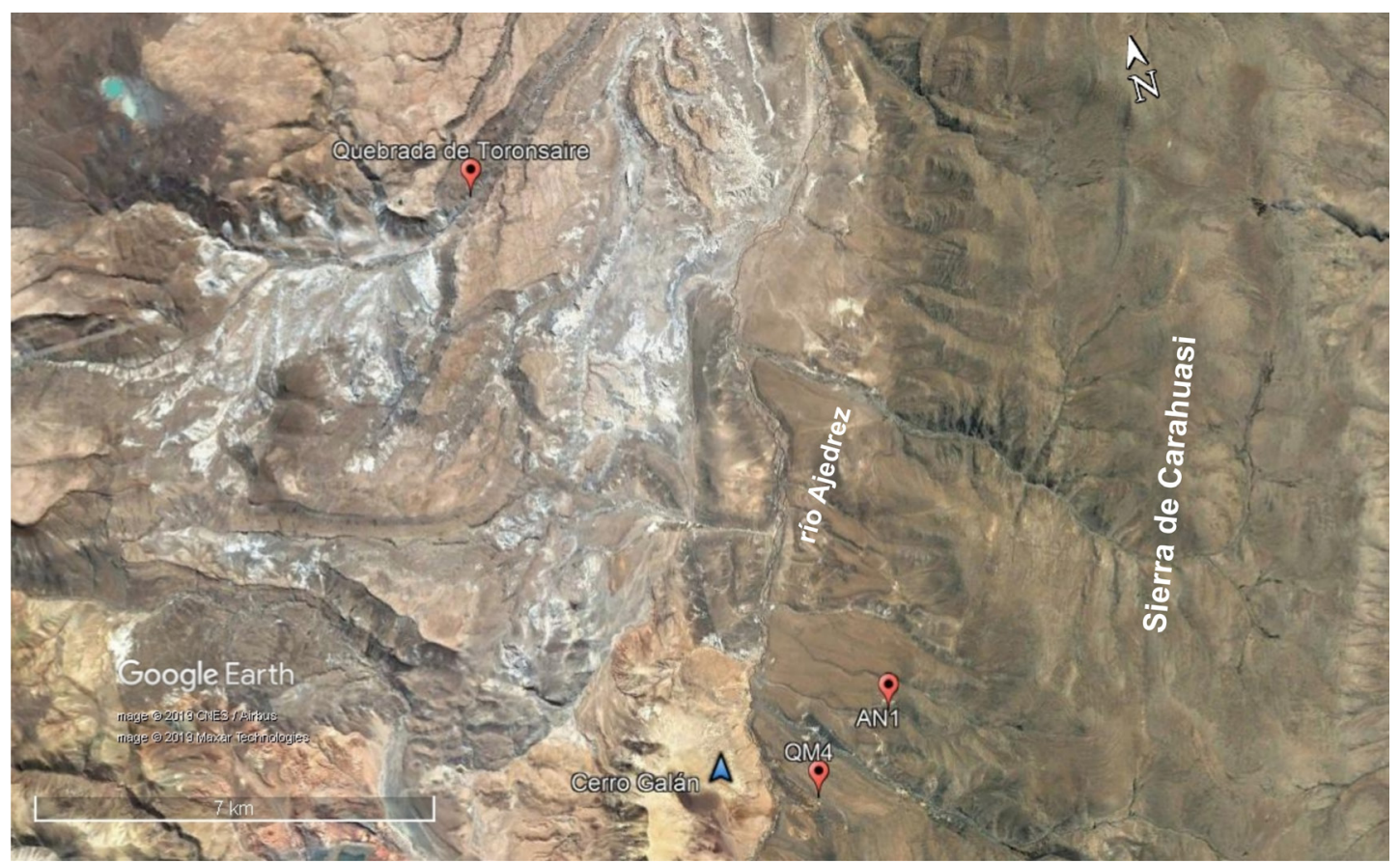

Figura 2. Ubicación de los lugares mencionados en el texto. Imagen satelital de Google Earth.

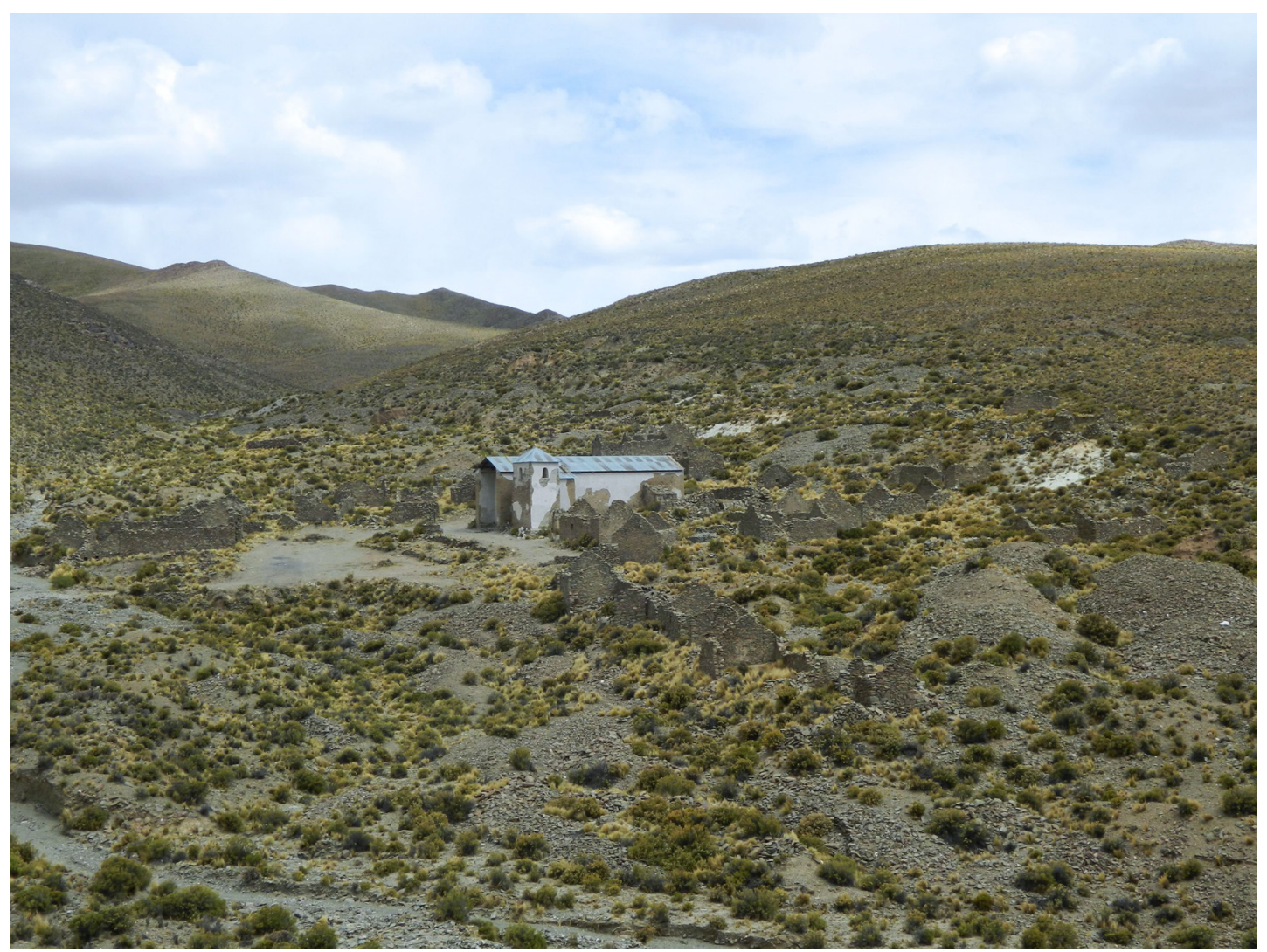

Figura 3. Conjuntos de estructuras de Antiguyoc vistos desde el cementerio que se ubica contiguo al poblado. Destaca en el centro la iglesia con su campanario y el espacio abierto enfrente a modo de "plaza". 


\section{Aproximación desde las fuentes escritas}

Hemos rastreado referencias a prácticas de manufactura, circulación y uso de recipientes dentro de la variada gama de documentos históricos relacionados o que aluden a la localidad de Antiguyoc (censos, padrones, juicios, testamento, libros de fábrica, libros de bautismos, entre otras). El relevamiento evidenció que estas prácticas y sus objetos vinculados no eran frecuentemente plasmados en la escritura, y menos aún de modo principal, sino que las alusiones se reducen a casos en los que se llevaron a cabo inventarios o menciones de bienes por algún motivo. Son los casos del testamento de 1776 de quien fuera uno de los propietarios de la hacienda de Antiguyoc (el español Fernando Dávalos) ${ }^{1}$, de la posterior adjudicación de bienes a sus acreedores en $1778^{2}$ $\mathrm{y}$ de un juicio llevado adelante por la muerte de un joven en $1780^{3}$, en el que se embargan los bienes de un indígena acusado del homicidio (Tabla 2). Algunos de estos documentos fueron trabajados antes desde investigaciones históricas y etnohistóricas con otros objetivos (cf. Gil Montero 2004; Sánchez 2002; Ulloa 2005).

En el caso del testamento de Dávalos, se consignan, entre otras, pertenencias vinculadas a objetos contenedores o utensilios de cocina. Los objetos son: una paila grande de cobre; dos pailas de cobre más pequeñas; un sartén de cobre y otra de hierro; seis "botijuelas bidriadas"; dos frascos de vidrio; tres platillos de peltre; una "limetilla vidriada" (conteniendo azogue), once barriles (nueve de ellos con agua y dos con aguardiente) y ollas (ubicadas en una cocina). No podemos dejar de señalar que las pailas y sartenes son declarados en su testamento pero no se encontraban en la vivienda al momento del inventario.

Dos años después de este documento, durante un juicio por la sucesión de los bienes de Dávalos y su adjudicación a los respectivos acreedores (septiembre-octubre de 1778) se mencionan objetos que habrían sido de su pertenencia, los que se encontraban en posesión del indio Pedro Mamani, entre ellos: "cuatro limetas de loza ordinaria"; "seis tinajas de hacer chicha, y un belero [sic] todo de barro todo biejo [sic] y quebrado" (f.7v); un frasco de vidrio quebrado" (f.10). ${ }^{4}$ Llama la atención que se mencionan bienes que al parecer no habían sido inventariados antes, como las limetas de loza. Asimismo, tal vez las tinajas de hacer chicha corresponden a las ollas que se habían mencionado antes, las que se encontraban ubicadas en la cocina.

A su vez, en la casa del indio Fausto Mamani se encontraron ocho cántaros; doce ollas de barro y tres "birques" (sic). En el caso de los cántaros y los virques se hace la aclaración que son "de hacer chicha". 5

\section{Análisis del material cerámico y sus resultados}

Los materiales cerámicos analizados hasta el momento $(\mathrm{N}=812)$ corresponden a una porción del material recolectado, pero nos permiten delinear algunas tendencias generales (Tabla 3). Identificamos un amplio predominio de las cerámicas rojas $(\mathrm{n}=709$, 87,3\%) que involucran tanto piezas abiertas (Figura 4) como cerradas (fundamentalmente vasijas y ollas y posiblemente cántaros) (Figura 5), con superficies alisadas. Se observan macrotrazas que se asocian al levantado por superposición de rollos de arcilla $(n=59)$, así como variaciones de espesor verticales, uniones de segmentos imperfectamente ensambladas y fracturas en facetas cúbicas o longitudinales. Otras se pueden vincular al estirado con los dedos $(n=6)$, como variaciones de espesor horizontales, depresiones circulares y marcas de dedos. Algunos fragmentos $(n=7)$ de asas de este tipo de recipientes presentan modelados al pastillaje o incisiones en zigzag. Los recipientes fueron cocidos en atmósferas oxidantes, incompletas o completas, a base del color de las fracturas y la presencia de núcleos.

Estos materiales presentan, en análisis con lupa binocular, pastas con componentes comparables a los de la cerámica local de otros sitios prehispánicos y coloniales del sur de Pozuelos (Pérez Pieroni 2015b), como inclusiones densas de mica, cuarzo, minerales félsicos y litoclastos sedimentarios, cuya importancia varía de un tipo de pasta a otro. Algunos fragmentos $(\mathrm{n}=7)$ de cerámicas rojas alisadas fueron reutilizados para elaborar objetos circulares tipo fichas, halladas mayormente en el CA $8(n=6)$ y un ejemplar en el CA 9.

En ocasiones $(n=57)$ los fragmentos de cerámicas rojas de todos los conjuntos analizados presentan manchas oscuras o depósitos de hollín que vinculamos con exposición al fuego. En los casos en los que se pudieron hacer apreciaciones acerca de su morfología $(n=7)$ se trata de recipientes cerrados de diferentes tamaños (con diámetros en la boca entre 11 y $36 \mathrm{~cm}$ ). Sin embargo, tanto 
Tabla 1. Muestra de fragmentos analizados para cada conjunto arquitectónico (CA)

\begin{tabular}{crrrrr}
\hline & Bordes & Bases & Asas & Cuerpo & TOTAL \\
\hline CA 1 & 8 & 3 & 3 & 68 & 82 \\
CA 2 & 19 & 13 & 9 & 111 & 152 \\
CA 3 & 9 & 8 & 1 & 117 & 135 \\
CA 4 & 5 & 4 & 2 & 34 & 45 \\
CA 5 & 9 & 3 & 3 & 51 & 66 \\
CA 6 & 13 & 2 & 9 & 97 & 129 \\
CA 7 & 5 & 1 & 0 & 50 & 53 \\
CA 8 & 3 & 4 & 0 & 22 & 54 \\
Plaza frente a iglesia & 8 & 1 & 3 & 40 & 34 \\
CA 9 & 6 & 0 & 1 & 10 & 50 \\
CA 10 & 1 & & & & 12 \\
\hline
\end{tabular}

Tabla 2. Fuentes escritas inéditas analizadas en este trabajo

\begin{tabular}{cccc}
\hline Fuente & Contenido & Fecha & Lugar \\
\hline $\begin{array}{c}\text { ATJ, Carpeta 50, } \\
\text { Legajo 1653 }\end{array}$ & $\begin{array}{c}\text { Testamento e inventario de } \\
\text { bienes del español Fernando } \\
\text { Dávalos }\end{array}$ & 1776 & $\begin{array}{c}\text { Vivienda de Dávalos ubicada en } \\
\text { Quebrada del Maray 4 }\end{array}$ \\
AHJ, Colección del Marqués, \\
Carpeta 57 & $\begin{array}{c}\text { Depósito de los bienes de } \\
\text { Fernando Dávalos y juicio } \\
\text { sucesorio }\end{array}$ & 1778 & $\begin{array}{c}\text { Vivienda de Dávalos ubicada en } \\
\text { Quebrada del Maray 4 }\end{array}$ \\
ATJ, Carpeta 54, & Juicio por la muerte de \\
Legajo 1756 & Pablo Cayo en Antiguyoc & 1780 & $\begin{array}{c}\text { Vivienda de Fausto Mamani } \\
\text { ubicada en la Quebrada de } \\
\text { Toronsaire }\end{array}$ \\
\hline
\end{tabular}

Tabla 3. Tipos de materiales cerámicos hallados en el material fragmentario de Antiguyoc 1

\begin{tabular}{cccccc}
\hline & Cerámicas rojas & $\begin{array}{c}\text { Cerámicas } \\
\text { vitrificadas }\end{array}$ & Loza & Porcelana & Gres \\
\hline CA 1 & 73 & 6 & 3 & 0 & 0 \\
CA 2 & 142 & 3 & 4 & 0 & 1 \\
CA 3 & 117 & 4 & 10 & 3 & 0 \\
CA 4 & 38 & 2 & 4 & 0 & 0 \\
CA 5 & 63 & 2 & 0 & 0 & 0 \\
CA 6 & 124 & 2 & 2 & 0 & 0 \\
CA 7 & 51 & 1 & 5 & 0 & 0 \\
CA 8 & 45 & 2 & 30 & 0 & 0 \\
Plaza frente a iglesia & 2 & 1 & 5 & 0 & 0 \\
CA 9 & 42 & 0 & 0 & 4 & 1 \\
CA 10 & 12 & 27 & 64 & & 0 \\
\hline
\end{tabular}

estas como otras trazas macroscópicas generadas por el uso son difíciles de registrar en conjuntos procedentes de recolecciones superficiales, que han estado expuestos a procesos de meteorización que contribuyen a obliterarlas o enmascararlas. Se registró erosión de bordes o de las superficies (incluyendo sus acabados, como pinturas, engobes y vitrificados) en 288 fragmentos. Probablemente sea el resultado de agentes de meteorización como el viento, el agua, o la circulación de personas que visitan la iglesia y el sitio en determinadas fechas del año (como se menciona más adelante), entre otros.

En menor medida se observan recipientes de cerámicas rojas con vitrificados en la superficie 


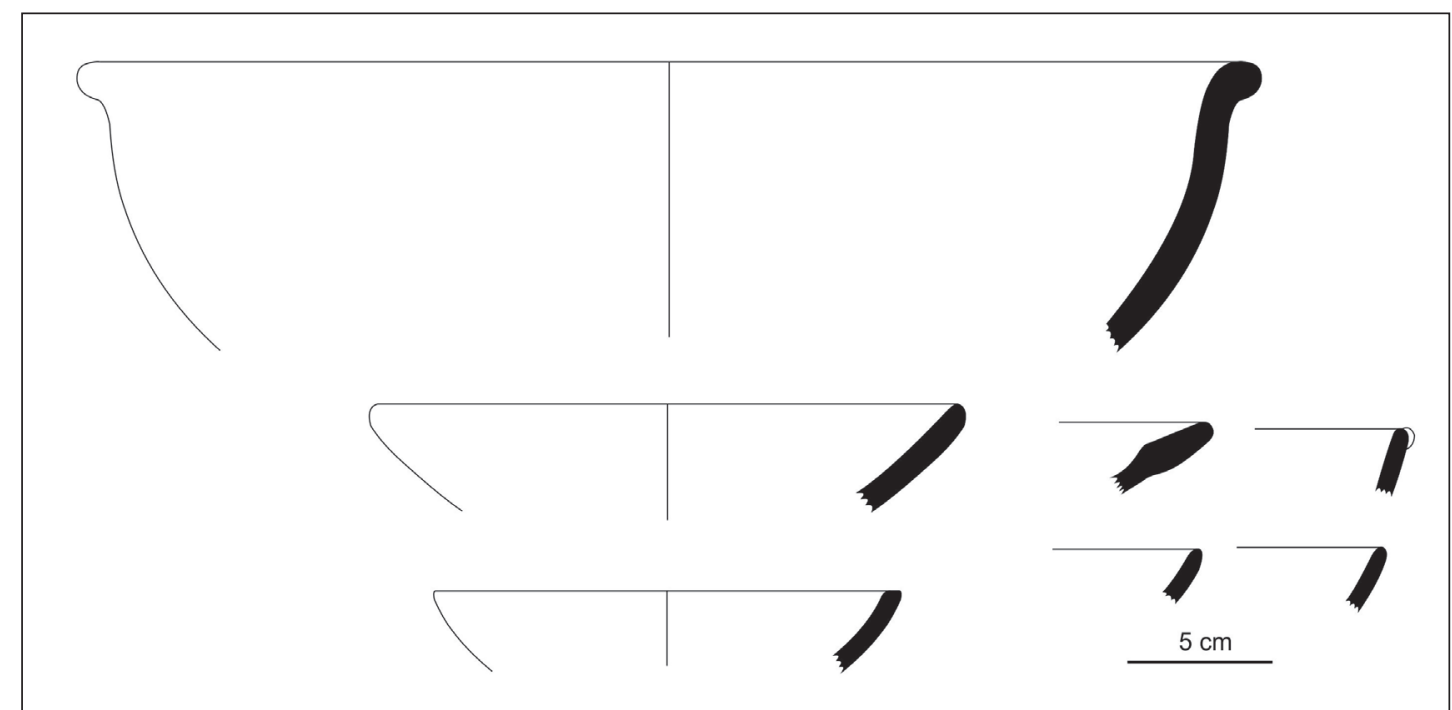

Figura 4. Reconstrucciones morfológicas de recipientes abiertos de cerámicas rojas con superficies alisadas recuperados en Antiguyoc.

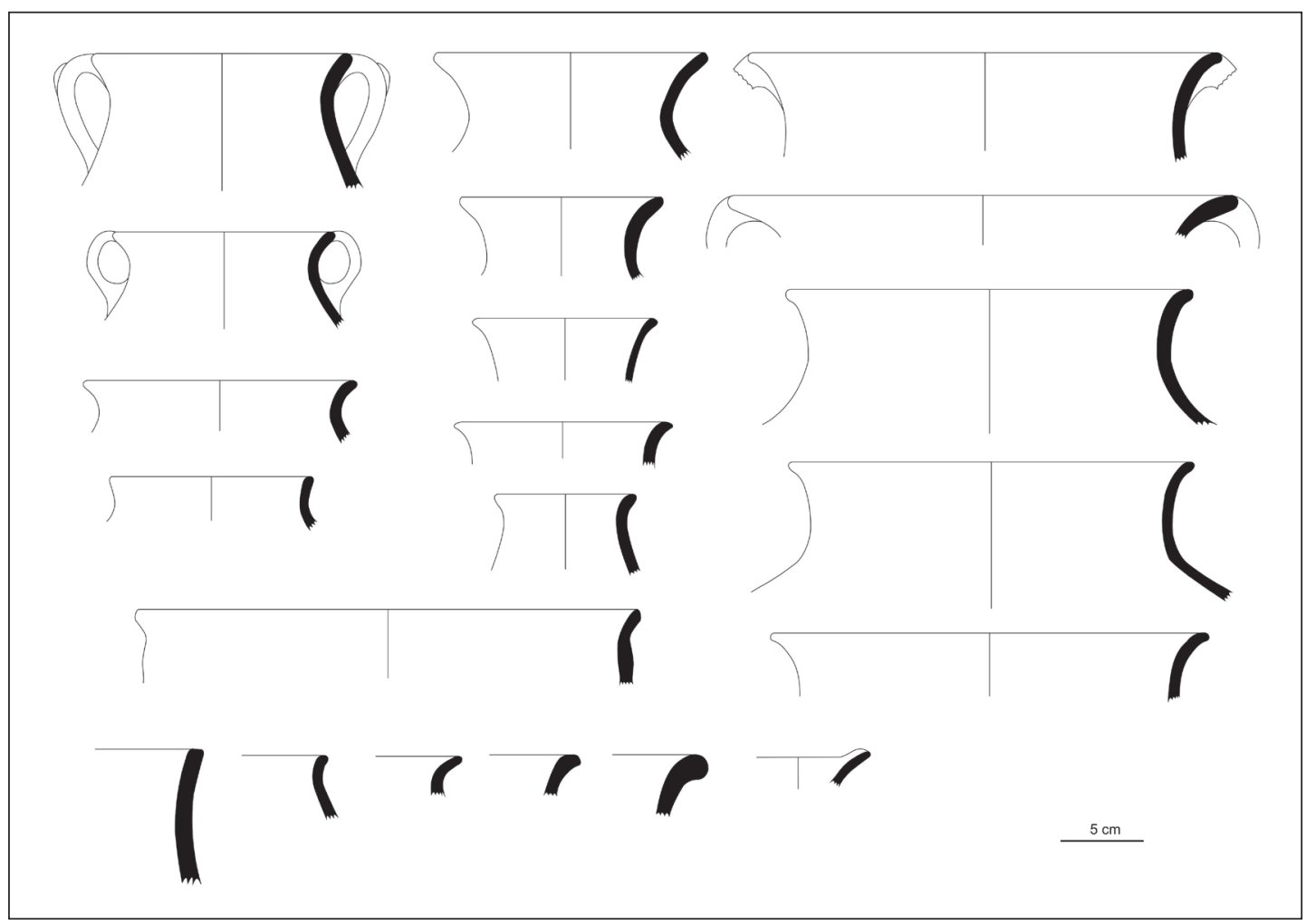

Figura 5. Reconstrucciones morfológicas de recipientes cerrados de cerámicas rojas con superficies alisadas recuperados en Antiguyoc.

interna $(n=27 ; 3,3 \%)$. Todos ellos corresponden a recipientes abiertos (Figura 6), y en algunos casos presentan evidencias de modelado con torno $(n=8)$, consistentes en ondulaciones horizontales y estrías alargadas y paralelas. Ocasionalmente, se observan motivos pintados cercanos al borde. En la Figura 7 se muestran algunos ejemplos de estos fragmentos.

También hemos registrado fragmentos de recipientes abiertos de loza ( $\mathrm{n}=64 ; 7,9 \%$ ) (Figura 8), correspondientes mayormente a los tipos whiteware 


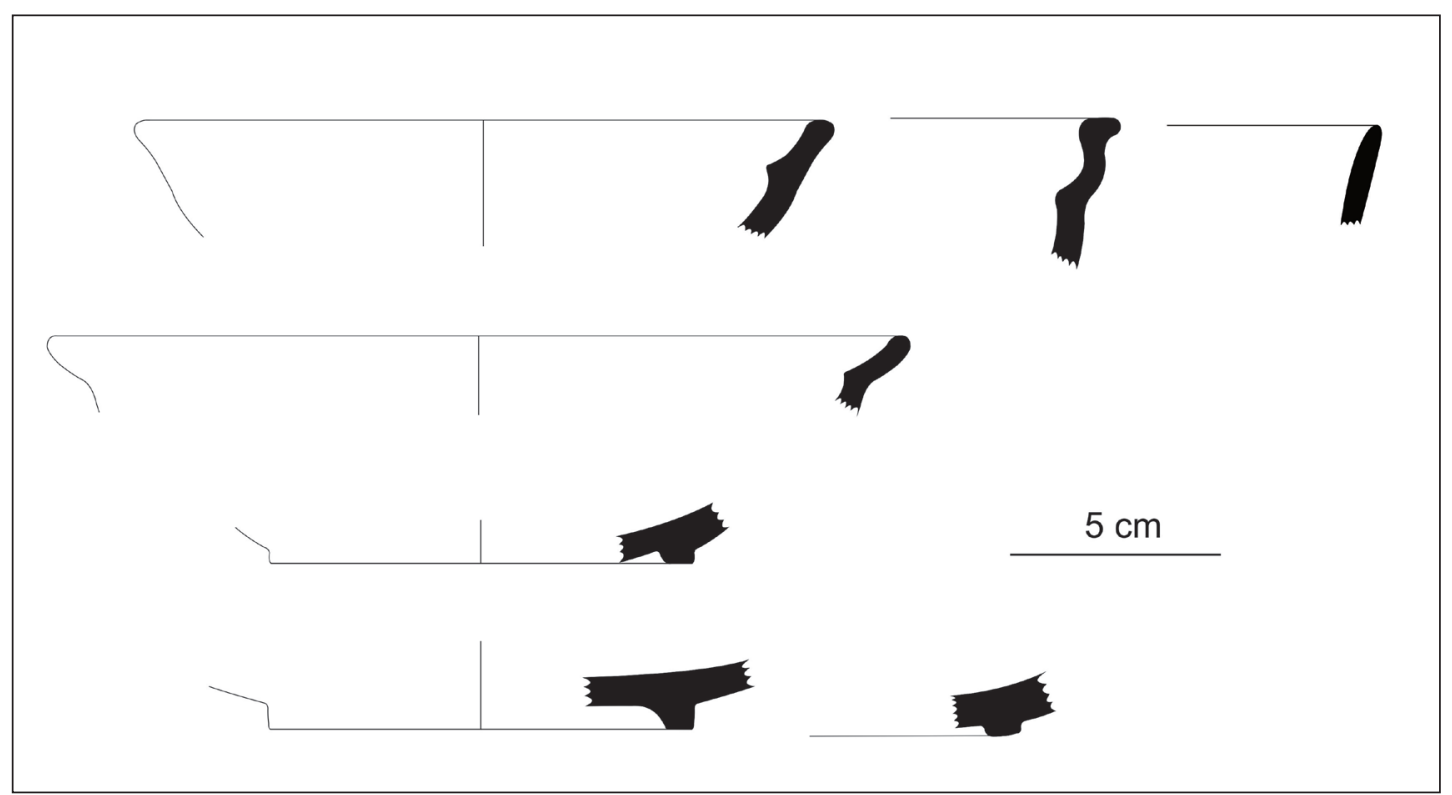

Figura 6. Reconstrucciones morfológicas de recipientes abiertos de cerámicas rojas con superficies vitrificadas recuperados en Antiguyoc.

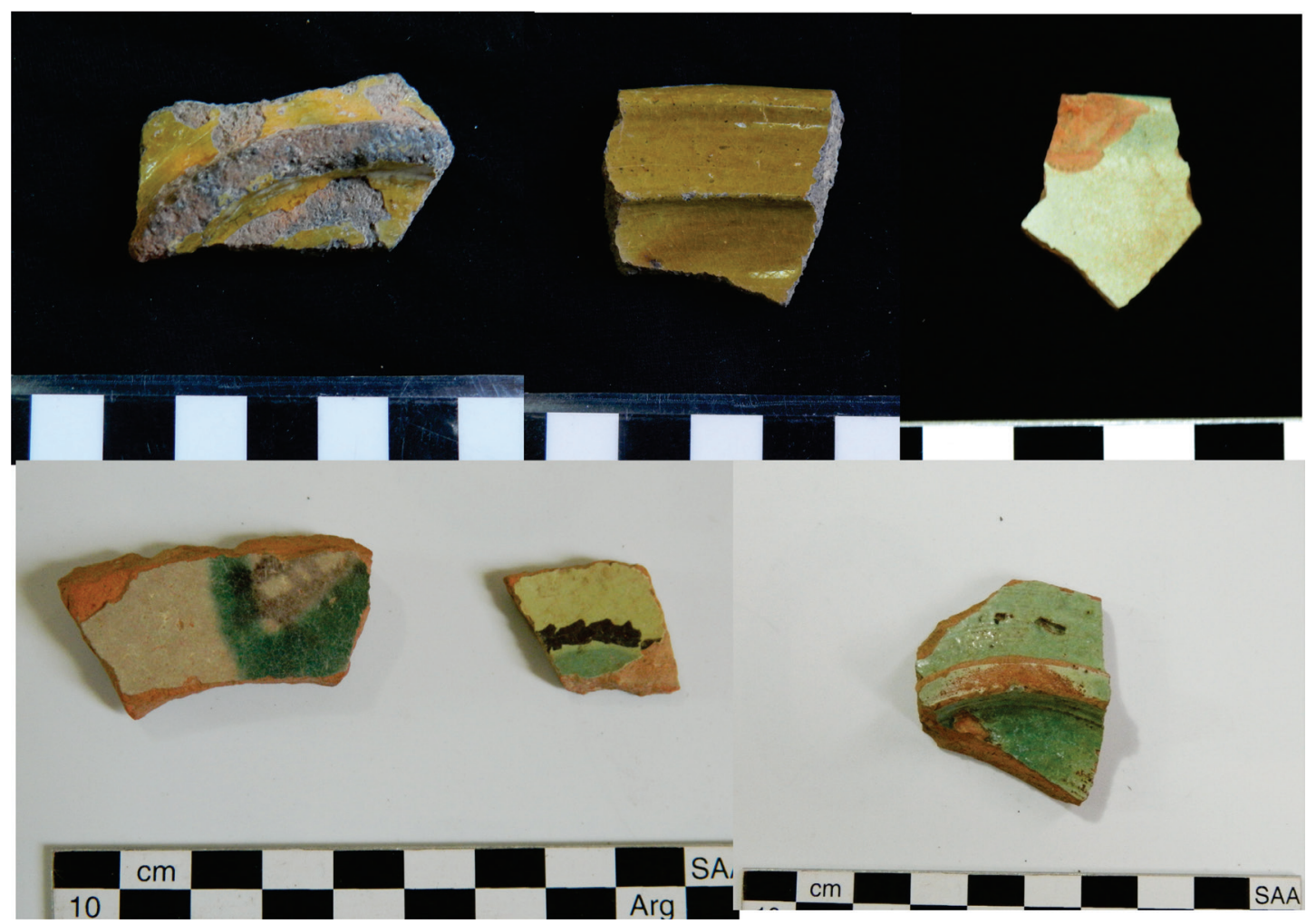

Figura 7. Ejemplos de fragmentos de cerámicas rojas con vitrificados recuperados en Antiguyoc. 
$(n=48)$ y en menor medida pearlware $(n=8)$ e indeterminados $(n=8)$, con motivos en diferentes colores, o sin pintura (Figura 9). Un número importante de estos fragmentos $(n=18)$, procedentes del espacio tipo plaza frente a la iglesia, corresponden a un mismo plato, que presenta anillos concéntricos en gris en la superficie interna.
Cuatro fragmentos son de porcelana, tres corresponden a tres recipientes diferentes del CA 3 y uno del CA 9, sin motivos pintados. Las morfologías y los fragmentos se ilustran en la Figura 10. Además, registramos un único fragmento de botella de gres. Ninguno de estos recipientes exhibe evidencias de exposición al fuego.

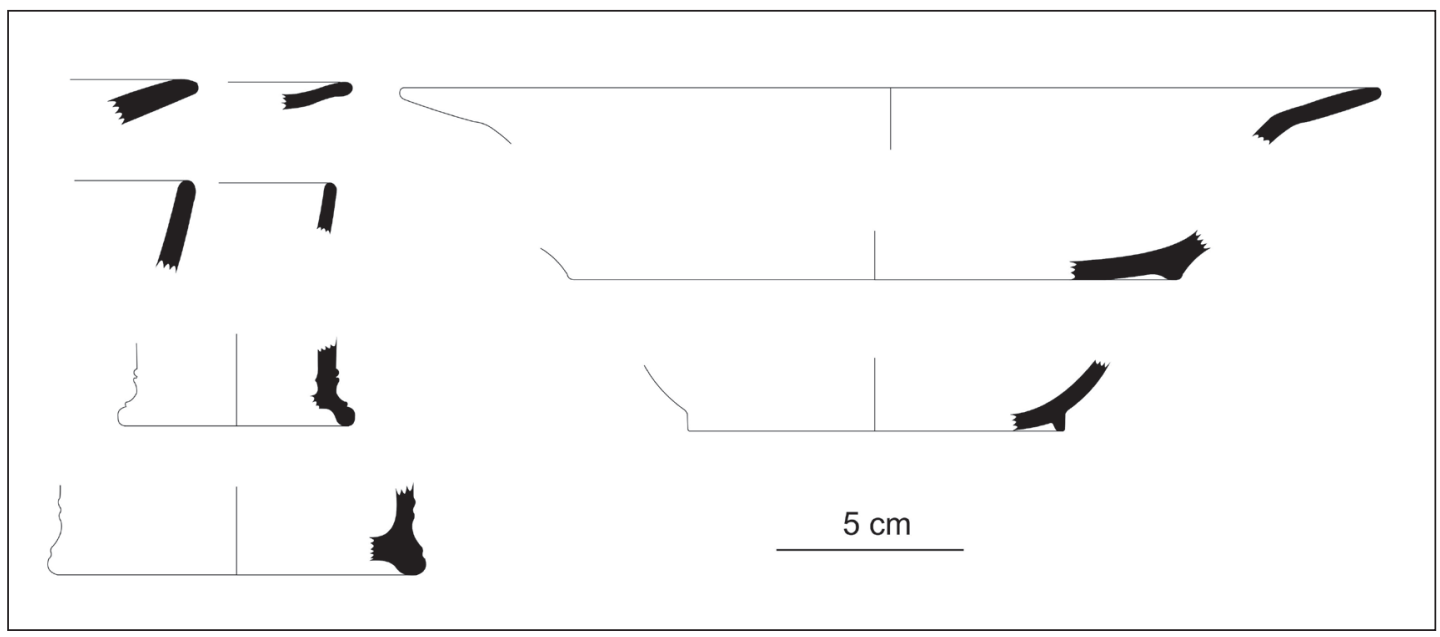

Figura 8. Reconstrucciones morfológicas de recipientes de loza recuperados en Antiguyoc.

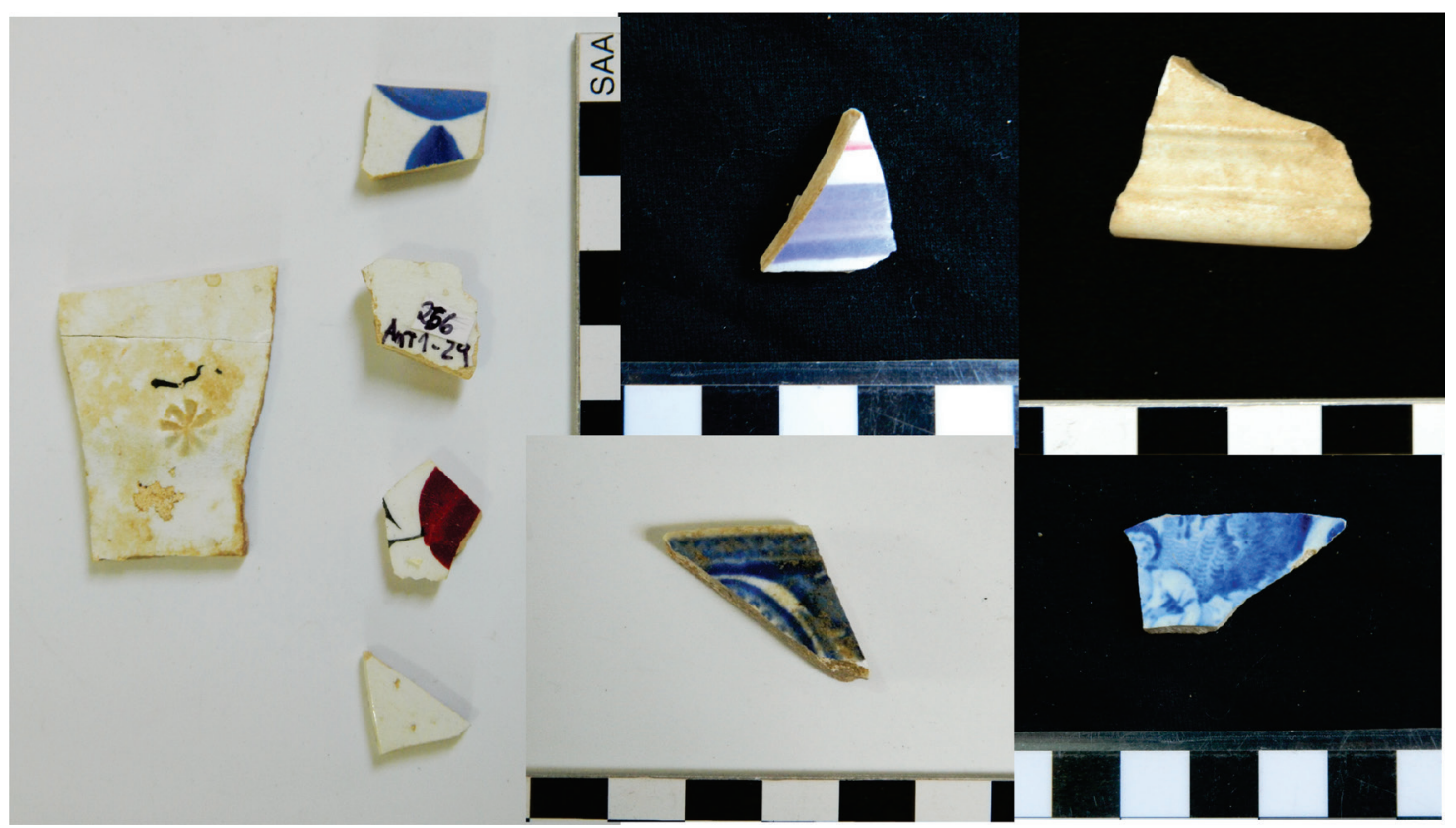

Figura 9. Ejemplos de fragmentos de loza recuperados en Antiguyoc. 


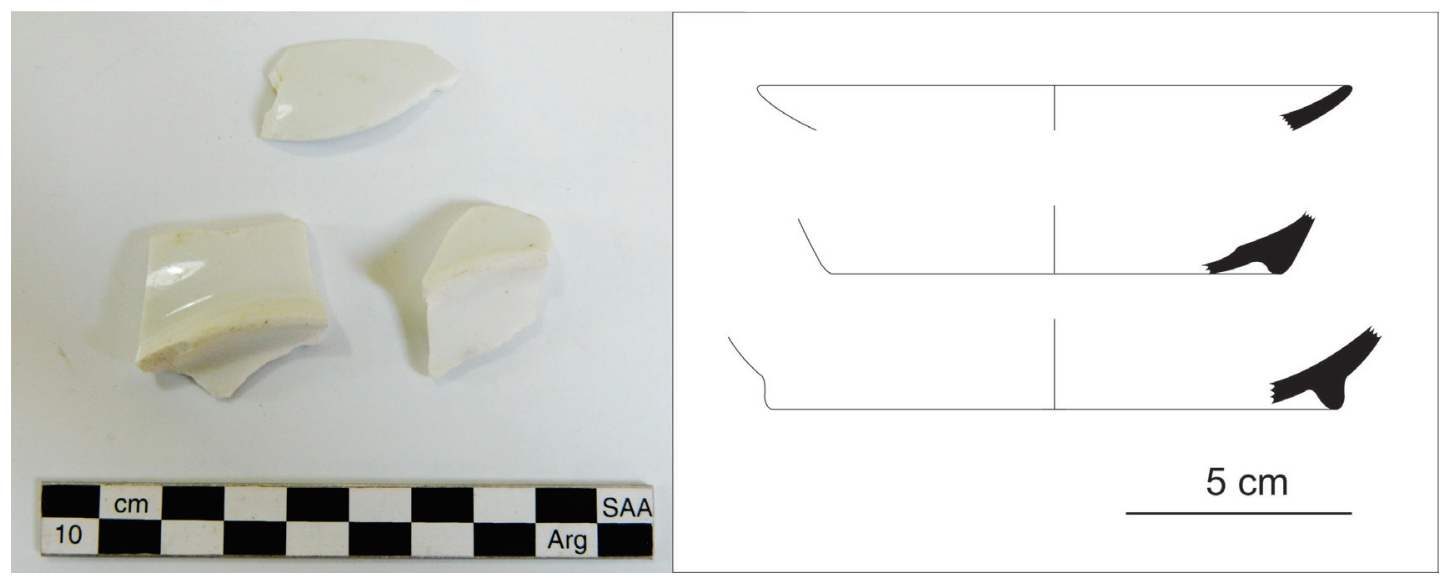

Figura 10. Fragmentos de porcelana de Antiguyoc y sus reconstrucciones morfológicas.

\section{Discusión}

Las características del material cerámico analizado hasta la fecha, particularmente en relación con los atributos vinculados con el modelado y las características de las pastas, nos permiten postular una continuidad en ciertas elecciones técnicas dentro de una tradición tecnológica regional que perdura con posterioridad a la llegada de los españoles. Sin embargo, se incorporan nuevos elementos, como motivos en zigzag sobre algunos fragmentos de asas, o los vitrificados en las superficies de algunos recipientes abiertos, que hemos mencionado previamente, y que probablemente fueron incorporados desde otras regiones. Estos materiales son comparables con los de otros sitios mineros coloniales de la Puna de Jujuy, como Pan de Azúcar o Timón Cruz, cuyas investigaciones hemos presentado en contribuciones anteriores (Pérez Pieroni 2018).

Asimismo, en Antiguyoc aparecen otros elementos que no habíamos registrado antes, como las lozas, porcelana y gres, que con seguridad tienen procedencias extrarregionales. Su baja cantidad en los conjuntos abordados puede estar evidenciando su escaso uso o alto valor, aunque las lozas pearlware y whiteware y el gres se popularizan en el siglo XIX (Schavelzon 2001), particularmente después de las guerras de independencia, cuando las nuevas naciones sudamericanas establecen lazos comerciales con Gran Bretaña (Brooks et al. 2019). Llama la atención la presencia más abundante de las lozas y porcelanas en el CA 3, que se localiza contiguo a la iglesia, y la gran cantidad de fragmentos de loza en el espacio abierto frente a la misma (aunque muchos pueden corresponder a un mismo recipiente fracturado allí). Esto estaría representando un sector del sitio con materiales más tardíos, tal vez producto de una ocupación más prolongada, no solo de los espacios de habitación sino también del sector externo inmediato, el que se vincula espacialmente de manera muy estrecha con la iglesia. Incluso en la actualidad este sector es utilizado por las personas que peregrinan al lugar durante fiestas religiosas ( 7 de octubre y 2 de noviembre por ejemplo), las que involucran actividades en la iglesia y en el espacio abierto frente a ella.

Es interesante destacar que estos materiales novedosos correspondan a recipientes de servicio (tazas, platos, escudillas, etc.) o, en menor medida, de almacenamiento de sustancias y bebidas (posible albarelo y botella de gres). Los recipientes empleados en la preparación de alimentos y posiblemente de bebidas (como por ejemplo la chicha, mencionada en las fuentes escritas) continuaron siendo las cerámicas rojas de producción local. Todos estos recipientes habrían sido parte de las actividades cotidianas de las familias que habitaban en el sitio, cuya densidad de construcciones permite pensar en una importante población para un paraje de la época en la Puna de Jujuy.

Respecto de la aproximación desde los documentos históricos, si bien las referencias de las que disponemos no son aún abundantes, cuentan con la ventaja de ser contemporáneas entre sí y de abarcar dos espacios dentro de una misma localidad, una hacienda española en un asiento de minas y una 
vivienda indígena en un sector "rural", los que son representativos de dos modos de habitar diferentes para una misma época.

En primer lugar, surgen diferencias entre las pertenencias detalladas en las viviendas española e indígena. Estas no radican tanto en la cantidad de elementos sino en la variedad, presentándose en la vivienda del propietario diversos elementos de cocina (muchos de estos son de metal) y otros contenedores. En cuanto a los materiales cerámicos, destacan recipientes cerrados descriptos como "vidriados", entre los que es interesante notar que uno de ellos ("limetilla") ${ }^{7}$ estaba destinado a contener azogue (mercurio) ${ }^{8}$, elemento utilizado en minería para el beneficio de la plata. Respecto de las "limetas de loza ordinaria", consideramos que se están refiriendo a recipientes de cerámicas rojas, quizás con vitrificado o esmaltado en sus superficies. Esto se sustenta en definiciones de la palabra como la que aportan Lister y Lister (1976: 57), quienes en su glosario definen "loza" como un término que se empleaba entre los siglos XIV y XVIII genéricamente para designar a las cerámicas, incluyendo la "loza común", "corriente" u "ordinaria", que involucra a las cerámicas rojas con o sin vitrificados, entre otras.

Desafortunadamente en el documento de 1776, cuando se mencionan las ollas de la cocina no se ofrecen más detalles, como tampoco su cantidad. En 1778 se indica la pertenencia de seis tinajas de hacer chicha, pero no podemos saber si se trata de los mismos elementos. De todas maneras, las referencias a pertenencias de Dávalos nos permiten sugerir que en la hacienda española también se utilizaban en la cocina contenedores de manufactura indígena. A su vez, en la vivienda del indio Mamani se mencionan cántaros, ollas y virques, objetos que a priori podemos asignar a las cerámicas rojas. Como señalamos antes, exceptuando las ollas, el resto de los recipientes habrían estado destinados a la elaboración de chicha. A la inversa que en el caso anterior, en esta vivienda no habrían contado con elementos de tradición europea. Esto encuentra coincidencias con los contextos arqueológicos coloniales que hemos excavado en áreas "rurales", en los que los materiales hallados son en todo coincidentes con aquellos de tradición prehispánica (Angiorama et al 2018b).

Las categorías morfológicas que mencionan los documentos son las mismas que registró Menacho $(2001,2007)$ (ollas, tinajas, virques, cántaros, entre otras) en unidades domésticas pastoriles actuales del departamento Rinconada, cuyas morfologías son comparables a las relevadas por nosotros como ollas, tinajas y posibles cántaros. Nielsen (2000) también relevó morfologías similares entre los pastores de Cerrillos (Lípez, Bolivia).

En la actualidad, estos pastores obtienen sus piezas de regiones próximas a nuestra área de estudio (Casira, Berque, etc.) y las emplean en diferentes actividades domésticas y rituales como preparación y consumo de alimentos y bebidas, higiene de diferentes elementos y alumbrado de habitaciones (Nielsen 2000; Menacho 2001, 2007). Incluso en aquellas comunidades que han incorporado el uso de recipientes de otros materiales, como metal o plástico, los recipientes cerámicos siguen siendo apreciados y empleados para la preparación de alimentos y son indispensables en la elaboración de chicha (Nielsen 2000; Menacho 2001).

De manera similar, pero para la Quebrada de Humahuaca, Cremonte et al. (2009) registran el uso de jarras, ollas, cántaros y virques para la elaboración de chicha en comunidades actuales de El Perchel.

En estos registros actuales, algunos de estos recipientes, relacionados con la preparación y servicio de chicha, son altamente valorados, conservados y cuidados, y llegan a tener antigüedades considerables (aproximadamente 50 años). En las actividades documentadas, se exponen al fuego ollas y algunos cántaros. Por su parte, los virques son los recipientes de mayor tamaño y con bocas de diámetros más grandes (45 cm aproximadamente, contra 25 de las ollas) (Menacho 2001; Cremonte et al. 2009).

Asimismo, Boman (1908), en sus exploraciones por la Puna a fines de siglo XIX, registra la producción y uso local de alfarería, distinguiendo recipientes de menores dimensiones $(20$ a $30 \mathrm{~cm}$ de diámetro) que se emplean en la elaboración de alimentos, y otros más grandes $(50$ a $60 \mathrm{~cm}$ de diámetro) empleados en la elaboración de la chicha.

En contribuciones anteriores (Angiorama et al. 2018a; Giusta 2019) hemos mencionado que la evidencia histórica sugiere que en un primer momento se emplearon indígenas de la encomienda de Casabindo y Cochinoca como mano de obra para las labores extractivas llevadas adelante en este asiento. En los años subsiguientes la fuerza de trabajo principal provino de los "forasteros sin tierra" residentes en la zona. Esto podría explicar la continuidad en la denominación de las morfologías 
mencionadas para la vivienda del indio Mamani y el contraste entre el uso de recipientes cerámicos en la vivienda indígena en comparación al uso de recipientes de diferentes materiales en la vivienda española. También daría cuenta de la continuidad que observamos en ciertas elecciones técnicas en la manufactura cerámica, en relación con materiales prehispánicos de la región, y del uso de la alfarería local, cuya diversidad de formas y tamaños relevados hasta ahora, junto con algunas evidencias de exposición al fuego, podrían estar indicando su perduración en usos tradicionales (como preparación de alimentos y bebidas tanto en la vida cotidiana como para festividades), así como se registra incluso hasta la actualidad.

\section{Conclusiones}

Para concluir, queremos destacar que el diálogo entre los registros materiales, escritos y la información etnográfica y etnoarqueológica resultó fructífero en el propósito de comenzar a analizar ciertas prácticas sociales del período colonial. Lejos de buscar correlaciones directas entre los datos obtenidos a partir de distintas evidencias, el abordaje desde campos disciplinares diversos nos permite contar con un panorama más amplio de la problemática que aquel que obtendríamos desde el análisis de una única fuente de datos. Para ello, debido a que la documentación vinculada a la Puna de Jujuy no suele hacer referencias directas a este tipo de prácticas cotidianas y su materialidad involucrada, es necesario abordarlas con una "mirada arqueológica" que permita rastrearlas dentro de escritos elaborados con propósitos diversos.

\section{Agradecimientos}

Las investigaciones que permitieron realizar este artículo contaron con el apoyo de subsidios PICT (FONCyT), PIP (CONICET) y PIUNT (Universidad Nacional de Tucumán). Queremos agradecer a los habitantes de la Puna en los distintos sectores en los que trabajamos y a los integrantes del equipo que han colaborado en diferentes instancias del trabajo de campo y laboratorio. También a las coordinadoras y a los participantes del simposio "TUKMA. Paisajes, textos, imágenes y materialidades (siglos XVI-XVIII)" del XX Congreso Nacional de Arqueología Argentina por la oportunidad de presentar una versión previa de este trabajo, por proporcionar un valioso espacio de discusión e intercambio que permitieron enriquecerlo y por la invitación a publicar en el presente Dossier. Finalmente, a los/las evaluadores por sus relevantes comentarios y sugerencias. Todo lo aquí vertido es, sin embargo, de nuestra responsabilidad.

\section{Referencias Citadas}

Angiorama, C. I., M. Giusta, M. F. Becerra y M. J. Pérez Pieroni. 2018a. “"La furia de buscar el oro'. Los asientos mineros del siglo XVIII y XIX en la Puna de Jujuy, Argentina”. Memoria Americana 26 (2): 8-26.

Angiorama, C., M. J. Pérez Pieroni, M. F. Becerra y M. Giusta 2018b. "Cambios y continuidades en la Puna de Jujuy (actual Argentina) durante la Colonia". Población \& Sociedad 25 (1): 5-43.

Bagaloni, V.

2017 "Aporte al estudio de materiales vítreos en contextos fronterizos y rurales: la casa de negocio Chapar (partido de Gonzales Chaves, provincia de Buenos Aires)". Intersecciones en Antropología 18 (1): 113-118.

Balfet, H; M. F. Fauvet-Berthelot y S. Monzón

1992 Normas para la Descripción de Vasijas Cerámicas. Centre D'Études Mexicaines et Centraméricaines, México D.F.

Brooks, A., S. Urbina, L. Adán, D. Carabias, V. Sepúlveda, H. Chiavazza y V. Zorrilla

2019 "The Nineteenth-Century British Ceramics Trade to Southwestern South America: An Initial Characterization of the Archaeological Evidence from Chile". En Archaeologies of the British in Latin America, editado por C. E. Orser Jr., pp. 55-71. Springer International Publishing, New York. Boman, E.

1908 Antiquités de la Région Andine de la République Argentine et du Désert d'Atacama. Tomo II. Librairie H. Le Soudier, Imprimerie Nationale, Paris.

Cremonte, M. B., C. Otero y M. S. Gheggi

2009 "Reflexiones sobre el consumo de chicha en épocas prehispánicas a partir de un registro actual en Perchel (Dto. Tilcara, Jujuy)". Relaciones de la Sociedad Argentina de Antropología XXXIV: 75-102.

García, M. S., M. F. Alonso, M. Auge, M. A. Moretti, J. Haidar y P. Martínez

2012 Empinando una limeta. Análisis del material vítreo del sitio El Santuario I, partido de Magdalena (Buenos Aires). En Actas del V Congreso Nacional de Arqueología Histórica (Tomo 2), editado por E. M. Rodríguez Leirado y D. Schávelzon, pp. 392-411. Editorial Académica Española. 
García Roselló, J. y Calvo Trías, M.

2013 Making Pots. El modelado de la cerámica y su potencial interpretativo. BAR International Series 2540. Oxford, Archaeopress.

Giusta, M.

2019 Modos de construir y habitar en la Puna de Jujuy. Un abordaje desde la arqueología histórica en la localidad de Antiguyoc (ca. 1774-1824), Provincia de Jujuy, Argentina. Tesis para optar por el grado de doctor, Facultad de Ciencias Naturales e IML, Universidad Nacional de Tucumán, Tucumán.

Gil Montero, R.

2004 Caravaneros y transhumantes en los Andes meridionales. Población y familia indígena en la puna de Jujuy. 1770-1870. Instituto de Estudios Peruanos, Lima.

Langiano, M. C., J. F. Merlo y V. Pedrotta

2009 "El patrimonio arqueológico de la antigua Frontera Sur: fuertes, fortines y tolderías". En Patrimonio, Ciencia y Sociedad. Un abordaje preliminar en los Partidos de Azul, Olavarría y Tandil, editado por J. Prado y M. Endere, pp. 235-258. UNICEN, Olavarría.

Lister, F. C. y R. H. Lister

1976 A Descriptive Dictionary for 500 Years of SpanishTradition Ceramics (13 th to $18^{\text {th }}$ Centuries). Special Publication Series $N^{\circ} 1$. The Society for Historical Archaeology.

Menacho, K.

2001 "Etnoarqueología de trayectorias de vida de vasijas cerámicas y modo de vida pastoril". Relaciones de la Sociedad Argentina de Antropología XXVI: 119-144.

Menacho, $\mathrm{K}$.

2007 "Etnoarqueología y estudios sobre funcionalidad cerámica: aportes a partir de un caso de estudio". Intersecciones en Antropología 8: 149-161.

Nielsen, A. E.

2000 Andean Caravans: an ethnoarchaeology. Tesis Doctoral inédita. Departamento de Antropología, Universidad de Arizona. MS.

Pérez Pieroni, M. J.

2015a. "Prácticas productivas y tradiciones tecnológicas: la manufactura cerámica prehispánica tardía y colonial en la cuenca sur de Pozuelos y el área de Santa Catalina, Puna de Jujuy, Argentina". Relaciones de la Sociedad Argentina de Antropología XL (1): 13-44.

Pérez Pieroni, M. J.

2015b. "La manufactura cerámica prehispánica tardía y colonial en la cuenca sur de Pozuelos y el área de Santa Catalina (Jujuy, Argentina): caracterización petrográfica de pastas cerámicas". Intersecciones en Antropología 16: 237-44.

Pérez Pieroni, M. J.

2018 "Tecnología cerámica de época colonial en la cuenca sur de Pozuelos y el área de Santa Catalina, Puna de Jujuy, Argentina". Revista de Arqueología Histórica Argentina y Latinoamericana 12: 116-140.

Real Academia Española

1780 Diccionario Academia Usual. http: //ntlle.rae.es/ntlle/ SrvltGUIMenuNtlle?cmd=Lema\&sec=1.0.0.0.0.

Schavelzon, D.

2001 Catálogo de cerámicas históricas de Buenos Aires (s. XVI-XX). [Formato CD]. Fundación para la Investigación del Arte Argentino (FIAAR), Buenos Aires.

Terreros y Pando, E. de

1767 Diccionario castellano con las voces de ciencias y artes y sus correspondientes en las tres lenguas francesa, latina e italiana, Tomo segundo. Madrid. Reproducido a partir del ejemplar de la Biblioteca de la Real Academia Española.

Ulloa, M.

2005 Comerciantes, pulperos, hacendados y buscadores de oro. Españoles en la Puna de Jujuy a fines del Siglo XVIII. Actas del VI Congreso Internacional de Etnohistoria. Buenos Aires, Argentina.

Vergara, M. A.

1942 Estudios sobre historia eclesiástica de Jujuy. Instituto de Historia, Lingüística y Folklore, Universidad Nacional de Tucumán. San Miguel de Tucumán.

Zaburlín, M. A.

2014 Uso, consumo y circulación de vasijas cerámicas en los pueblos prehispánicos de la laguna Guayatayoc (Puna de Jujuy). Tesis para optar por el grado de doctor, Facultad de Ciencias Naturales e IML, Universidad Nacional de Tucumán, Tucumán.
1 Archivo de Tribunales de Jujuy (ATJ), Carpeta 50, Legajo 1653, año 1776.

2 Archivo Histórico de Jujuy (AHJ), Colección Ricardo Rojas, Carpeta 57, año 1778.

3 ATJ, C.54, Leg. 1756, año 1780, f. 6.

4 Respecto de los recipientes de vidrio, si bien no es el tema de este trabajo, corresponde señalar que las fuentes escritas de 1776 y 1778 mencionan algunos frascos de este material, mas no así botellas. ¿Circularían botellas de vidrio en Antiguyoc en esta época? La respuesta que por el momento podemos ofrecer desde la arqueología es negativa, ya que de los análisis preliminares que hemos realizado acerca de fragmentos diagnósticos del material vítreo recolectado en Antiguyoc 1 no surgieron elementos que pudieran indicar su fabricación antes de 1822 (fecha en la que comienza a utilizarse el molde de dos partes o molde Ricketts).

5 ATJ, C.54, Leg. 1756, año 1780, f. 6.

6 Con este término hacemos referencia a que, según lo que se desprende del documento, la vivienda habría consistido en un enclave de producción y reproducción de la unidad doméstica similar a los actuales "domicilios" o "estancias" de pastoreo (sensu Tomasi 2010), es decir, que no se encontraba en un asiento de mina o en un poblado con intervención estatal colonial, como una parroquia o viceparroquia.

7 Considerando la terminología de la época, se trataría de un recipiente "a modo de redoma", es decir, de cuerpo curvo 
y cuello largo (cf. Real Academia Española, Diccionario Academia Usual 1780: 584,3; Terreros y Pando 1787: $456,1)$. En cambio, en estudios de casos correspondientes a mediados y fines del siglo XIX el término se usa para nombrar botellas troncopiramidales que contenían principalmente ginebra (cf. Langiano et al., 2009; García et al., 2012; Bagaloni 2017, entre otros).

8 ATJ, C.50, Leg. 1653, año 1776 (sin foliar). 